\title{
Rare Cause of Severe Neurological Impairment in a Young Patient With Lupus
}

\author{
Bogdan Sorohan ${ }^{\mathrm{a}, \mathrm{c}}$, Andreea Gamala ${ }^{\mathrm{a}}$, Marina Paraschiv ${ }^{\mathrm{a}}$, Roxana Jurubita ${ }^{\mathrm{a}, \mathrm{b}}$, \\ Ismail Gener ${ }^{a}, b$
}

\begin{abstract}
Systemic lupus erythematous (SLE) is a chronic autoimmune disease that frequently leads to kidney involvement. Hypertension in lupus nephritis (LN) is common, but malignant hypertension (MH) is a rare condition. $\mathrm{MH}$ in $\mathrm{LN}$ is associated with severe clinical manifestations and intrarenal vascular lesions. A 19-year-old woman with a history of SLE, class VI LN and MH presented with sudden drop in general status, confusion, diplopia, bradypsychia, and bradylalia. Physical exam showed cold extremities, cyanosis, high blood pressure $(260 / 100 \mathrm{~mm} \mathrm{Hg})$ and tachycardia. Neurological exam showed impairment of the cerebellum, left central facial palsy and left-sided hemiparesis. Laboratory work showed thrombocytopenia, hyperuricemia, hypoalbuminemia and an estimated glomerular filtration rate of $66.1 \mathrm{~mL} / \mathrm{min} / 1.73 \mathrm{~m}^{2}$. A cerebral computed tomography (CT) showed no hemorrhagic lesions, but a diffuse hypodense edematous area in the pons, midbrain and bilateral thalamic nuclei. She was started on antihypertensive therapy, dexamethasone and mannitol. After 5 days in intensive care unit, a brain magnetic resonance angiogram (MRA) showed petechial hemorrhage and swelling in deep thalamic regions, left internal capsule, pons, and midbrain. Nimodipine was added to the regimen. Workup for JC virus and Listeria monocytogenes was negative. Evolution was favorable with complete remission of neurological symptoms. Repeated brain MRA confirmed regression of swelling and hemorrhagic lesions. The diagnosis of hypertensive encephalopathy was sustained by improved clinical evolution under anti-edema measures, antihypertensives and by $\mathrm{CT} /$ magnetic resonance imaging (MRI). Differential diagnosis was made with central nervous system vasculitis and infections due to immunosuppression therapy (tuberculosis, HIV, and JC virus). This case describes the evolution of a young woman with lupus and recurrent $\mathrm{MH}$, complicated with neurological manifestations.
\end{abstract}

Keywords: Systemic lupus erythematosus; Lupus nephritis; Malignant hypertension; Neurological manifestations; Hypertensive encephalopathy

Manuscript accepted for publication October 17, 2016

aDepartment of Nephrology, Fundeni Clinical Institute, Bucharest, Romania ${ }^{\mathrm{b} C}$ arol Davila University of Medicine and Pharmacy, Bucharest, Romania ${ }^{c}$ Corresponding Author: Bogdan Sorohan, Department of Nephrology, Fundeni Clinical Institute, Bucharest, Romania. Email: bogdan.sorohan@yahoo.com

doi: http://dx.doi.org/10.14740/jmc2676w

\section{Introduction}

Systemic lupus erythematous (SLE) is a chronic autoimmune disease with unknown etiology that usually affects young women, with a wide range of clinical manifestations and laboratory findings. Pediatric SLE is different from the adult form, because of its longer disease activity, with a greater renal, cardiovascular (hypertension) and neurological involvement [1]. Incidence of hypertension in lupus nephritis (LN) is variable (34.3-42\%) and the incidence of malignant hypertension (MH) is only $7.6 \%$, associated with severe clinical manifestations and intrarenal vascular lesions [2]. Prevalence of hypertension is higher in women with SLE, even in those with non-advanced renal disease and chronic inflammation is considered a central pathway in renal vascular lesions and renal dysfunction [3]. MH was described in patients with antiphospholipid syndrome, primary or secondary to SLE [4]. MH with hypertensive retinopathy as onset of SLE was previously described in literature [5]. We report the case of a young female with SLE and $\mathrm{LN}$ who presented with recurrent $\mathrm{MH}$ and hypertensive encephalopathy.

\section{Case Report}

A 19-year-old woman with a history of SLE, class VI LN and MH presented with sudden drop in general status, confusion, diplopia, bradypsychia, and bradylalia. Her past medical history was notable for $\mathrm{MH}(300 / 120 \mathrm{~mm} \mathrm{Hg})$ complicated with hypertensive encephalopathy, seizures and acute respiratory distress syndrome that needed mechanical ventilation, at the age of 14. Three months later, a similar episode occurred and at that moment endocrine causes of hypertension were excluded. She was also diagnosed with left ventricular hypertrophy, mild diastolic dysfunction, mixed anxiety-depressive disorder and iron deficiency anemia. At the age of 15, she was diagnosed with mixed connective tissue disease based on clinical manifestations (sausage-shaped fingers, Raynaud phenomenon, capillary dilation and avascular areas at nail fold capillaroscopy), serological findings (positivity for antinuclear antibodies and anti-U1-RNP (263 IU/mL), and negativity for anti-dsDNA) and imaging (lung fields with infiltrates, with ground glass changes; pericardial effusion). Azathioprine was started. Subsequently, at the following ad- 

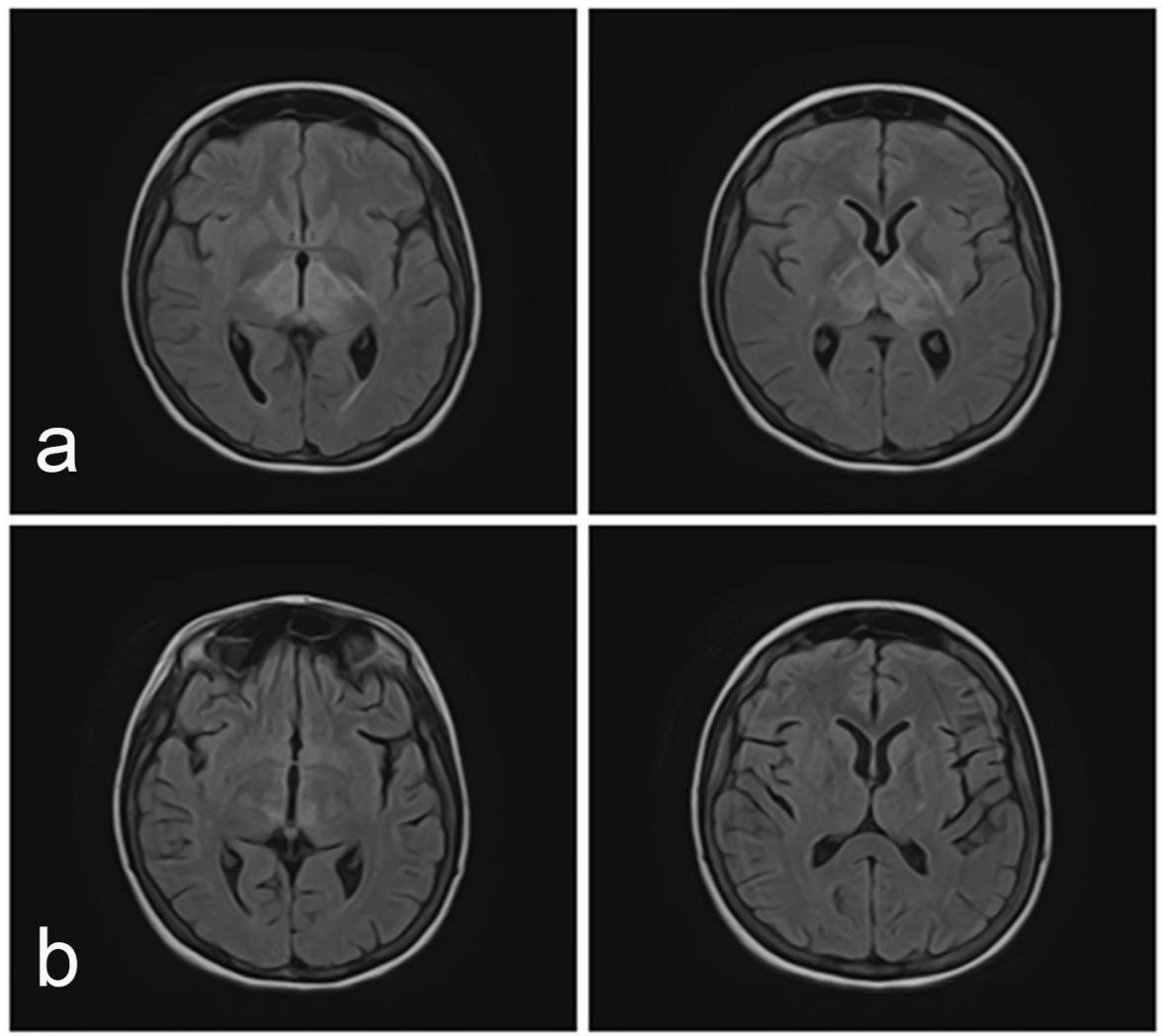

Figure 1. Axial MRI images (FLAIR sequence). (a) Hyperintense signal in deep white and grey matter, suggestive of vasogenic edema, with atipical localization involving bilateral deep talamic regions (more pronounced on the left side), pontomesencephalic region and left internal capsule. (b) After 5 days follow-up, MRI shows resolution of previously lesions.

mission, the diagnosis was reevaluated and SLE was considered based on clinical features, immunology (at this time anti-dsDNAs were positive), renal involvement, psychiatric manifestations and serositis. Pulse therapy with methylprednisolone and cyclophosphamide was started, followed by oral prednisone with slowly tappering dose until the current admission. Hydroxychloroquine was later added to the regimen. At the age of 18, a renal biopsy was performed, showing class VI LN with positive ANA speckled pattern, glomerulosclerosis, and hypertensive nephrosclerosis. At admittance the patient was afebrile, with no edema, severe confusion, cold extremities, cyanosis, raised blood pressure (260/100 $\mathrm{mm} \mathrm{Hg})$, tachycardia (100 bpm), with an oxygen saturation of $89 \%$. Heart sounds were normal and no crackles were present. Neurological exam showed impairment of the cerebellum, left central facial palsy and left-sided hemiparesis. Laboratory work showed mild thrombocytopenia $\left(105,000 / \mathrm{mm}^{3}\right)$, hyperuricemia $(11.2 \mathrm{mg} / \mathrm{dL})$, serum creatinine of $1.19 \mathrm{mg} / \mathrm{dL}$ with an estimated glomerular filtration rate (eGFR) of $66.1 \mathrm{~mL} / \mathrm{min} / 1.73 \mathrm{~m}^{2}$ and urea of $89.0 \mathrm{mg} / \mathrm{dL}$. On ECG, a sinus rhythm, with a rate of $130 \mathrm{bpm}$, a normal QRS axis $\left(0^{\circ}\right)$, poor $\mathrm{R}$ wave progression, voltage criteria of left ventricular hypertrophy, and flattened $\mathrm{T}$ waves in $\mathrm{aVL}$ and V1 were found. A non-enhanced cerebral computed tomography (CT) showed no hemorrhagic lesions, but with a diffuse hypodense area in the pons, midbrain and bilateral thalamic nuclei. She was started on antihypertensive therapy, dexamethasone and mannitol. Neurological status continued to deteriorate with tetraplegia and a GCS of 8, so the patient was transferred to the intensive care unit (ICU). A lumbar puncture excluded meningitis: increased protein level (1.79 $\mathrm{g} / \mathrm{L})$, decreased glucose level $(2.3 \mathrm{mmol} / \mathrm{L}), 3 \mathrm{WBC} / \mu \mathrm{L}$ with $100 \%$ monocytes, $0 \mathrm{RBC} / \mu \mathrm{L}$, negative gram stain and in microscopy three leukocytes, and four RBC. Also, workup for $\mathrm{JC}$ virus and Listeria monocytogenes was negative. After 5 days, a brain angio-MRI (MRA) was performed and showed petechial hemorrhage and signal abnormalities suggestive for swelling lesions in deep thalamic regions, left internal capsule, pons, and midbrain. Nimodipine was added to the regimen. Evolution was favorable with complete remission of neurological symptoms. Repeated head MRA confirmed regression of swelling and hemorrhagic lesions (Fig. 1). The patient was discharged after 17 days, with good general status and a therapeutic regimen of mycophenolate mofetil, hydroxychloroquine and antihypertensive therapy.

\section{Discussion}

This case describes the evolution of a young woman with LN and recurrent $\mathrm{MH}$, complicated with neurological manifestations. The three episodes of $\mathrm{MH}$ were associated with neuro- 
logical impairment and admittance to the ICU. Based on past history, clinical features, laboratory and imagistic findings, a differential diagnosis for sudden cognitive decline was necessary. Central nervous system (CNS) lupus is a life-threatening condition and CNS vasculitis was excluded through CT and especially MRI. Also, a brain biopsy could have been performed [6]. Other causes of neurological impairment in lupus are infections (meningitis and encephalitis) due to immunosuppression treatment: tuberculosis, HIV, JV virus, that produce progressive multifocal leukoencephalopathy. Our patient presented negative workup for all of these. The diagnosis of MH complicated with hypertensive encephalopathy was sustained by improved clinical evolution under anti-edema measures (mannitol and dexamethasone), antihypertensive therapy and by CT/MRI. A particularity of this case is the location of the cerebral lesions, not a common parietal-occipital pattern that is associated with PRES. Another is the absence of antiphospholipid syndrome (APS), $\mathrm{MH}$ being more common in SLE patients with APS compared with SLE patients without APS [7]. In conclusion, we describe a case of a young patient with $\mathrm{LN}$ and recurrent $\mathrm{MH}$, complicated with neurological manifestations. $\mathrm{MH}$ is an important cause of neurological manifestations in SLE with LN, other than cerebral vasculitis and infections.

\section{Acknowledgments}

We would like to thank Drs. Mihaela Gherghiceanu and Eugen Mandache for their interpretation of biopsies and Dr. Mihai Ranete for assistance with obtaining the CT/MRI digital im- ages.

\section{References}

1. Chan PC, Yu CH, Yeh KW, Horng JT, Huang JL. Comorbidities of pediatric systemic lupus erythematosus: A 6-year nationwide population-based study. J Microbiol Immunol Infect. 2016;49(2):257-263.

2. Yu SH, Whitworth JA, Kincaid-Smith PS. Malignant hypertension: aetiology and outcome in 83 patients. Clin Exp Hypertens A. 1986;8(7):1211-1230.

3. Ryan MJ. The pathophysiology of hypertension in systemic lupus erythematosus. Am J Physiol Regul Integr Comp Physiol. 2009;296(4):R1258-1267.

4. Cacoub P, Wechsler B, Piette JC, Beaufils H, Herreman G, Bletry O, Godeau P. Malignant hypertension in antiphospholipid syndrome without overt lupus nephritis. Clin Exp Rheumatol. 1993;11(5):479-485.

5. Choe JY, Park SH, Kim JY, Jung HY, Kim SK. A case of systemic lupus erythematosus presenting as malignant hypertension with hypertensive retinopathy. Korean J Intern Med. 2010;25(3):341-344.

6. Rowshani AT, Remans P, Rozemuller A, Tak PP. Cerebral vasculitis as a primary manifestation of systemic lupus erythematosus. Ann Rheum Dis. 2005;64(5):784-786.

7. Jouquan J, Pennec Y, Mottier D, Youinou P, Cledes J, Leroy JP, Le Menn G. Accelerated hypertension associated with lupus anticoagulant and false-positive VDRL in systemic lupus erythematosus. Arthritis Rheum. 1986;29(1):147. 